\title{
Chromatographic Study of Rutherfordium (Element 104) in the System HCl/Tributylphosphate (TBP)
}

\author{
By R. Günther, W. Paulus, J. V. Kratz, A. Seibert, P. Thörle, S. Zauner \\ Institut für Kernchemie, Universität Mainz, D-55099 Mainz, Germany
}

W. Brüchle, E. Jäger, V. Pershina, M. Schädel, B. Schausten

Geselischaft für Schwerionenforschung, D-64220 Darmstadt, Germany

D. Schumann

Institut für Analytische Chemie, TU Dresden, D-01062 Dresden, Germany

\author{
B. Eichler, H. W. Gäggeler, D. T. Jost and A. Türler
}

Paul Scherrer Institute, CH-5253 Villigen, Switzerland

(Received September 19, 1997; accepted November 10, 1997)

\section{Element 104 / Rutherfordium / \\ Extraction chromatography with tributylphosphate}

\begin{abstract}
Summary
Liquid-liquid extractions of ${ }^{95} \mathrm{Zr}$ and ${ }^{175} \mathrm{Hf}$ from various concentrations of $\mathrm{HCl}$ were conducted using tributylphosphate (TBP) in toluene (benzene), and undiluted TBP. Quantitative extraction of both $\mathrm{Zr}$ and $\mathrm{Hf}$ into TBP in toluene (benzene) was observed from $\geq 8 \mathrm{M} \mathrm{HCl}$ and into undiluted TBP from $\geq 10 \mathrm{M} \mathrm{HCl}$. Extraction from $8 \mathrm{M} \mathrm{HCl}$ into undiluted TBP was $80 \%$ for $\mathrm{Zr}$ and $20 \%$ for Hf. Based on these results, a chromatographic separation of $\mathrm{Hf}$ from $\mathrm{Zr}$ with the Automated Rapid Chemistry Apparatus ARCA II was performed on $1.6 \times 8 \mathrm{~mm}$ columns containing undiluted TBP on an inert support. This separation was also studied on-line with short-lived ${ }^{169} \mathrm{Hf}$ produced in the $\mathrm{Gd}\left({ }^{18} \mathrm{O}, \mathrm{xn}\right)$ reaction and with $78-\mathrm{s}{ }^{261} \mathrm{Rf}$ produced in the ${ }^{248} \mathrm{Cm}\left({ }^{18} \mathrm{O}, 5 \mathrm{n}\right)$ reaction at the Philips Cyclotron of the Paul Scherrer Institute. From the distribution of $\alpha$-decays of ${ }^{261} \mathrm{Rf}$ between the $\mathrm{Hf}$ - and $\mathrm{Zr}$-fraction, the $K_{d}$-value for $\mathrm{Rf}$ in $8 \mathrm{M} \mathrm{HCl}$ was determined, and the following extraction trend was established: $\mathrm{Zr}>\mathrm{Rf}>\mathrm{Hf}$. This is at variance with earlier results where $\mathrm{Rf}$ seemingly did not extract as well as $\mathrm{Hf}$ which was tentatively attributed to increased hydrolysis of $\mathrm{Rf}$.
\end{abstract}

\section{Introduction}

Tributylphosphate (TBP) is one of the most important organic extractants. It is widely used to separate $U$ and Pu from spent nuclear fuel $[1,2]$ and to separate various actinides and lanthanides from reprocessing wastes [3]. The phosphoryl oxygen coordinates with the metal ion forming an adduct:

$$
\mathrm{M}^{\mathrm{z}+}+\mathrm{z} \mathrm{A}^{-}+x \mathrm{TBP} \rightarrow \mathrm{MA}_{\mathrm{z}} \cdot x \mathrm{TBP}_{\text {org }}
$$

where $\mathrm{M}^{2+}$ is the metal ion and $\mathrm{A}^{-}$is the deprotonated form of the acid. TBP always extracts the metal in the form of a neutral species. The distribution coefficient, $K_{d}$, is defined as the ratio of the concentration of the metal in the organic phase to the metal concentration in the aqueous phase at equilibrium. Because of aggre- gation of the TBP, $K_{d}$ values for undiluted TBP are found to be relatively lower than for TBP diluted by an organic solvent which was recognized in the development of the PUREX process [4].

Early experiments on the aqueous chemistry of element 104, rutherfordium, Rf, showed that its chemical properties are analogous to those of the group 4 elements $\mathrm{Zr}$ and $\mathrm{Hf}[5,6]$ and that a new transition element series filling the $6 d$ shell starts with Rf. Silva et al. [5] demonstrated this by eluting Rf from cationexchange columns with $0.1 \mathrm{M} \alpha$-hydroxyisobutyrate at $\mathrm{pH}=4.0$ within the first few column volumes while trace quantities of the $3^{+}$actinide ions did not elute in over 100 column volumes. Hulet et al. [6] used the known chloride complexing of the group 4 elements to extract $\mathrm{Rf}$ anionic chlorides from $12 \mathrm{M} \mathrm{HCl}$ into columns loaded with trioctylmethylammonium chloride, i.e. under conditions where the actinides are not appreciably extracted. $\mathrm{Zr}$, $\mathrm{Hf}$, and $\mathrm{Rf}$ were subsequently eluted in $6 \mathrm{M} \mathrm{HCl}$, in which anionic chloride complexation of the group- 4 elements is less favored.

More recently, Czerwinski et al. [7] corroborated the anionic chloride complexation of $\mathrm{Rf}$ in $12 \mathrm{M} \mathrm{HCl}$ by liquid-liquid extractions into $0.1 \mathrm{M}$ triisooctylamine in benzene. They also performed a series of liquidliquid extractions with TBP/benzene [8] to study the effects of $\mathrm{HCl}-$, chloride-, and hydrogen-ion concentration between 8 and $12 \mathrm{M}$ on the extraction of $\mathrm{Zr}^{4+}$, $\mathrm{Hf}^{4+}, \mathrm{Th}^{4+}, \mathrm{Pu}^{4+}$, and $\mathrm{Rf}^{4+}$. It was found that $\mathrm{Rf}$ extraction increases as a function of $\mathrm{HCl}$ concentration and that the extraction of $\mathrm{Rf}$ is between that of $\mathrm{Zr}$ and Hf. In experiments in which the chloride and hydrogen ion were varied, Rf extraction differed from that of the group-4 elements and behaved more like $\mathrm{Pu}^{4+}$ [8].

Recently, Bilewicz et al. [9] studied the sorption of $\mathrm{Zr}, \mathrm{Hf}, \mathrm{Th}$, and $\mathrm{Rf}$ on cobalt ferrocyanide surfaces and interpreted the results such that the hydrolysis trend in these elements decreases in the order $\mathbf{R f}>$ 
$\mathrm{Zr}=\mathrm{Hf}>\mathrm{Th}$. This is in contradiction with expectations based on the simple ionic model of hydrolysis, for details see Sect. 4. This unexpected trend was attributed [9] to relativistic effects in $\mathrm{Rf}$ which split the $6 d$ orbitals into two $6 d_{3 / 2}$ orbitals and three strongly destabilized $6 d_{5 / 2}$ orbitals. The destabilization of the $6 d_{5 / 2}$ orbitals was believed to cause the promotion energy involving $7 s, 7 p$, and $6 d_{5 / 2}$ orbitals to be too high for $s p^{3} d^{4}$ hybridization. This was believed [9] to give $\mathrm{Rf}$ a coordination number of 6 rather than 8 in aqueous solutions because 6 -coordination requires only $6 d_{3 / 2}$ orbitals while 8-coordination also requires the $6 d_{5 / 2}$ orbitals. A coordination number of 6 would result in a larger hydrolysis constant than otherwise expected. We shall argue against this interpretation in the discussion. Kacher et al. [10] performed some additional chloride extractions into TBP/benzene with $\mathrm{Zr}$, Hf, and Ti. The reported low extraction yields of $\mathrm{Hf}$ by Czerwinski $e t$ al. [8] could not be reproduced by Kacher et al. [10] who reported that they observed that significant amounts of Hf (more than 50\% in some cases) stick to teflon surfaces. (They actually conducted their subsequent experiments with polypropylene equipment because only negligible absorption was observed with polypropylene surfaces.) The Hf results from the Czerwinski et al. experiments [8] were based on on-line data taken at the Cyclotron where the activity was collected on teflon discs which, according to [10], accounts for the seemingly low extraction. Surprisingly, a similar loss due to absorption of $\mathrm{Rf}$ in the Czerwinski et al. work [8] was not suspected by Kacher et al., and so the latter authors, based on their new Zr-, Hf-, and Ti-results and on the old [8] Rf results, suggested a revised sequence of extraction into TBP/benzene from around $8 \mathrm{M} \mathrm{HCl}$ as $\mathrm{Zr}>\mathrm{Hf}>\mathrm{Rf}>\mathrm{Ti}$. In a parallel study of liquid-liquid extractions of these elements into TBP/benzene from $\mathrm{HBr}$ solutions, extraction of Rf was found to be low and was only observed when $\mathrm{LiBr}$ was added to increase the bromide concentration beyond $9 \mathrm{M}[10]$. The extraction behaviour of the group- 4 elements into TBP from both $\mathrm{HCl}$ and $\mathrm{HBr}$ solutions was primarily attributed to their different tendencies to hydrolyze [10].

In hydrofluoric acid solutions, on-line chromatographic studies by Szeglowski et al. [11] indicated that $\mathrm{Rf}$ forms anionic fluoride complexes which sorb on anion-exchange columns. Kacher et al. [12] found extraction of $\mathrm{Rf}$ into triisooctylamine/xylene from $0.5 \mathrm{M}$ HF and claimed that the extractability in this system decreases in the order $\mathrm{Ti}>\mathrm{Zr}=\mathrm{Hf}>\mathrm{Rf}$ in inverse order of the decrease in ionic radii.

The present study of Rf extraction into TBP from $\mathrm{HCl}$ solutions was performed in view of the somewhat unsatisfactory situation with the conflicting $\mathrm{Hf}$ results in $[8,10]$ and with the intention to establish an independent set of data characterizing the extraction sequence of $\mathrm{Zr}, \mathrm{Hf}$, and $\mathrm{Rf}$ at $8 \mathrm{M} \mathrm{HCl}$. This particular concentration was chosen because, as will be enlarged below, the largest differences in extraction between $\mathrm{Zr}$ and $\mathrm{Hf}$ are observed here, so that a chromatographic separation of $\mathrm{Zr}$ and $\mathrm{Hf}$ appeared to be feasible which would allow us to determine the centroid of the elution position of $\mathrm{Rf}$ relative to that of $\mathrm{Zr}$ and $\mathrm{Hf}$.

\section{Experimental}

\section{A. Materials}

The TBP and other chemicals were purchased from Merck and used as received. Their purity was analytical grade (p.a.). Voltalef ${ }^{\mathrm{TM}}$ powder from Lehmann \& Voss, Hamburg, was used as inert support for the TBP column material. A range of particle sizes between 30 and $80 \mu \mathrm{m}$ was selected by sieving. These particles were coated with TBP, weight ratio Voltalef/TBP 5:1, by immersing the Voltalef in a solution of the TBP in cyclohexane and by removing the cyclohexane by evaporation under stirring.

\section{B. Tracer solutions}

The isotopes ${ }^{95} \mathrm{Zr}$ and ${ }^{175} \mathrm{Hf}$ were used as tracers. Carrier free ${ }^{95} \mathrm{Zr}$ was purchased from CIS Diagnostik $\mathrm{GmbH}$, Dreieich, and was delivered in $0.5 \mathrm{M}$ oxalic acid. This solution was evaporated to dryness in the presence of $\mathrm{H}_{2} \mathrm{O}_{2}$ and $\mathrm{HCl}$ several times to destroy the oxalic acid. It was then stored in $12 \mathrm{M} \mathrm{HCl}$ in a polypropylene flask. ${ }^{175} \mathrm{Hf}$ was obtained as decay product of ${ }^{175} \mathrm{~W}$ that was produced by $68 \mathrm{MeV} \alpha$-particle bombardment of a stack of thin Hf targets on $1.5 \mu \mathrm{m}$ $\mathrm{Al}$ backings at the Cyclotron of the Forschungszentrum Karlsruhe. The Al backings containing the reaction products were dissolved in conc. $\mathrm{HCl}$ and the $\mathrm{Al}$ was removed on an anion-exchange column (Dowex 1x8, $200 \mathrm{mesh}$ ). The ${ }^{175} \mathrm{Hf}$ was eluted in $7 \mathrm{M} \mathrm{HCl}$, evaporated to dryness, dissolved in $12 \mathrm{M} \mathrm{HCl}$, and stored in a polypropylene flask.

\section{Isotopes produced on-line}

\section{1. ${ }^{261}$ Rf production}

The isotope ${ }^{261} \operatorname{Rf}\left(t_{1 / 2}=78 \mathrm{~s}\right.$ [13]) was produced via the ${ }^{248} \mathrm{Cm}\left({ }^{18} \mathrm{O}, 5 \mathrm{n}\right)$ reaction at the Philips Cyclotron of the Paul Scherrer Institut, Villigen. The beam energy on target was $101 \mathrm{MeV}$ and the ${ }^{18} \mathrm{O}^{5+}$ beam intensity varied between 0.8 and $2.0 \mu \mathrm{A}$ (electrical). The target material, $\mathrm{Cm}_{2} \mathrm{O}_{3}$, isotopic composition $95.7 \%$ ${ }^{248} \mathrm{Cm}$, and $4.2 \%{ }^{246} \mathrm{Cm}$, was prepared by electrodeposition of $\mathrm{Cm}\left(\mathrm{NO}_{3}\right)_{3}$ in isopropanol on a $2.34 \mathrm{mg} / \mathrm{cm}^{2}$ $\mathrm{Be}$ foil by electrodeposition and by converting the nitrate to the oxide by heating the target to $600^{\circ} \mathrm{C}$. The target thickness was determined by $a$-particle counting to be $450 \mu \mathrm{g} / \mathrm{cm}^{2}$ of ${ }^{248} \mathrm{Cm}$. The products of the ${ }^{18} \mathrm{O}$ bombardment were transported to the chemistry apparatus by a helium $\mathrm{KCl}$ gas-jet transportation system at a flow rate of $1.6 \mathrm{l} / \mathrm{min}$.

\section{2. ${ }^{169} \mathrm{Hf}$ production}

The isotope ${ }^{169} \mathrm{Hf}\left(t_{1 / 2}=3.25 \mathrm{~min}\right)$ was produced via the $\mathrm{Gd}\left({ }^{18} \mathrm{O}, \mathrm{xn}\right)$ reaction at the Philips cyclotron using 
the same beam energy as above. The target material, $\mathrm{Gd}_{2} \mathrm{O}_{3}$, isotopic composition $26.2 \%{ }^{152} \mathrm{Gd}, 9.8 \%{ }^{154} \mathrm{Gd}$, $22.3 \%{ }^{155} \mathrm{Gd}, 16.5 \%{ }^{156} \mathrm{Gd}, 8.3 \%{ }^{157} \mathrm{Gd}, 10.2 \%{ }^{158} \mathrm{Gd}$, $6.7 \%{ }^{160} \mathrm{Gd}$, was prepared by electrodeposition as above. ${ }^{169} \mathrm{Hf}$ was transported to the chemistry apparatus by the same gas-jet transportation system for online tracer experiments under identical conditions as in the rutherfordium experiments. The ${ }^{169} \mathrm{Hf}$ also served to determine the gas-jet transportation yield by implanting it into a Au catcher foil placed directly behind the target and comparing its gamma activity with that at the collection site. The transport yield was $36 \%$.

\section{3. ${ }^{98} \mathrm{Zr}$ production}

${ }^{98} \mathrm{Zr}\left(t_{1 / 2}=30.7 \mathrm{~s}\right)$ was produced at the Mainz TRIGA reactor by thermal neutron induced fission of ${ }^{235} \mathrm{U}$. The $787 \mathrm{keV}$ gamma radiation of the daughter ${ }^{98 \mathrm{~B}} \mathrm{Nb}\left(t_{1 / 2}=\right.$ $2,9 \mathrm{~s})$ was detected which decays in transient equilibrium with ${ }^{98} \mathrm{Zr}$. ${ }^{98} \mathrm{Zr}$ was transported to the chemistry apparatus by a gas-jet transportation system and studied under identical conditions as in the rutherfordium experiments.

\section{Radiation detection}

Gamma-emitting isotopes were assayed using a highpurity $\mathrm{Ge}$ spectrometer system. $\alpha$-emitting ${ }^{261} \mathrm{Rf}$ was assayed with passivated ion-implanted planar silicon (PIPS) detectors calibrated with a ${ }^{212} \mathrm{~Pb}$ source milked from ${ }^{228} \mathrm{Th}$. The detection efficiency for $\alpha$-particles was $33 \%$. The time, channel number, and detector number of each incoming event was stored by a list processor driven by the GOOSY data acquisition and analysis software.

\section{E. ${ }^{95} \mathrm{Zr}$ and ${ }^{175} \mathrm{Hf}$ liquid-liquid extractions}

Liquid-liquid extractions of the $\mathrm{Zr}$ and $\mathrm{Hf}$ tracers were performed with TBP in toluene (benzene) in the volume ratio $1: 1(1.8 \mathrm{M})$ with $\mathrm{HCl}$ concentrations ranging from 1 to $12 \mathrm{M} .2 \mathrm{ml}$ of the organic phase were contacted with $2 \mathrm{ml}$ aqueous phase containing the tracer activity by shaking for $1 \mathrm{~min}$ in polypropylene tubes. Subsequently, $1 \mathrm{ml}$ of the aqueous and $1 \mathrm{ml}$ of the organic phase were removed and counted for activity. From the counts of the organic phase and the aqueous phase the $K_{d}$ or the percent extraction were obtained. It was verified that the sum of the counts was consistent with the initial activity. In order to study extractions of the tracers into undiluted TBP, $43 \mathrm{mg}$ of the TBP/Voltalef $(1: 5)$ powder $(7.39 \mu$ l of pure TBP) was contacted with $2 \mathrm{ml}$ of the aqueous phase by shaking for $1 \mathrm{~min}$. Here, a $1 \mathrm{ml}$ aliquot of the aqueous phase was counted to determine the $K_{d}$ which was calculated by taking the ratio of final activity over the initial activity.

\section{F. Extraction chromatography}

Reversed-phase extraction chromatography was performed on $1.6 \times 8 \mathrm{~mm}$ columns packed with TBP/Vol-

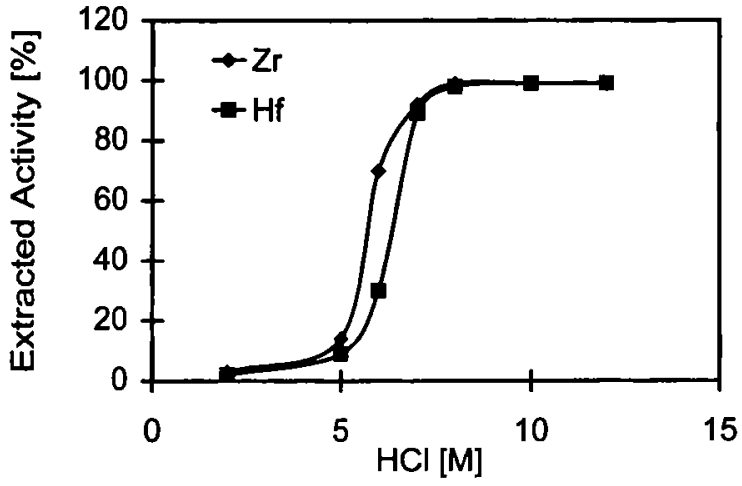

Fig. 1. Percent extraction of $\mathrm{Zr}$ and $\mathrm{Hf}\left({ }^{95} \mathrm{Zr}\right.$ and $\left.{ }^{175} \mathrm{Hf}\right)$ from $\mathrm{HCl}$ solutions into $\mathrm{TBP} /$ toluene $(1: 1)$.

talef $(1: 5)$ in the automated HPLC apparatus ARCA II [14]. The flow rate of the aqueous phase was $1 \mathrm{ml} /$ min. The columns were perconditioned with $200 \mu \mathrm{l}$ of $12 \mathrm{M} \mathrm{HCl}$. Originally, the tracers ${ }^{95} \mathrm{Zr}$ and ${ }^{175} \mathrm{Hf}$ were fed onto the column in $180 \mu \mathrm{l} 12 \mathrm{M} \mathrm{HCl}$ from a sample loop. In subsequent on-line experiments with ${ }^{98} \mathrm{Zr}$ at the TRIGA reactor and with ${ }^{169} \mathrm{Hf}$ and ${ }^{261} \mathrm{Rf}$ at the Philips cyclotron, the activity was deposited in ARCA II with the $\mathrm{KCl}$ aerosol by impaction, dissolved and fed onto the column in $167 \mu \mathrm{l} 12 \mathrm{M} \mathrm{HCl}$. This was followed by the elution of a $\mathrm{Hf}-$ and a $\mathrm{Zr}$ fraction in $200 \mu \mathrm{l}$ of $8 \mathrm{M} \mathrm{HCl}$ and $167 \mu \mathrm{l}$ of $2 \mathrm{M} \mathrm{HCl}$, respectively. These fractions were evaporated to dryness on Ta discs and assayed for gamma or $\alpha$-activity.

\section{Results}

Our liquid-liquid extraction data for ${ }^{95} \mathrm{Zr}$ and ${ }^{175} \mathrm{Hf}$ from $\mathrm{HCl}$ solutions into TBP/toluene $(1: 1)$ are shown in Fig. 1 . In contrast to the data by Czerwinski et al. [8], we find that $\mathrm{Hf}$, like $\mathrm{Zr}$, is completely extracted from $\geq 8 \mathrm{M} \mathrm{HCl}$. As Czerwinski et al. used TBP/benzene for their extractions, we checked the extraction data for ${ }^{95} \mathrm{Zr}$ and ${ }^{175} \mathrm{Hf}$ also with TBP/benzene $(1: 1)$ from $8 \mathrm{M} \mathrm{HCl}$; these data are indistinguishable from the TBP/toluene data for $8 \mathrm{M} \mathrm{HCl}$ in Fig. 1. Our data qualitatively corroborate the results by Kacher et al. [10], who report about $85 \%$ extraction of $\mathrm{Hf}$ and nearly complete extraction of $\mathrm{Zr}$ from $7.5 \mathrm{M} \mathrm{HCl}$ into $0.35 \mathrm{M}$ TBP/benzene. The Hf data by Czerwinski et al. [8] are obviously falsified by absorption.

As our goal was to use undiluted TBP/Voltalef for the chromatographic study of the sequence of extraction of $\mathrm{Zr}, \mathrm{Hf}$, and $\mathrm{Rf}$, we also determined the percentage extraction and the $K_{d}$-values of $\mathrm{Zr}$ and $\mathrm{Hf}$ with undiluted TBP/Voltalef $(1: 5)$ in batch experiments, see Fig. 2. As compared to the TBP/toluene (benzene) data, nearly complete extraction of both $\mathrm{Zr}$ and $\mathrm{Hf}$ is shifted to higher $\mathrm{HCl}$ molarities as expected, i.e. to $\geq 10 \mathrm{M} \mathrm{HCl}$, and there is a large difference in the percentage extraction of $\mathrm{Zr}(80 \%)$ and $\mathrm{Hf}(20 \%)$ at $8 \mathrm{M} \mathrm{HCl}$ which might give a chance for a chromatographic separation of $\mathrm{Zr}$ and $\mathrm{Hf}$. 


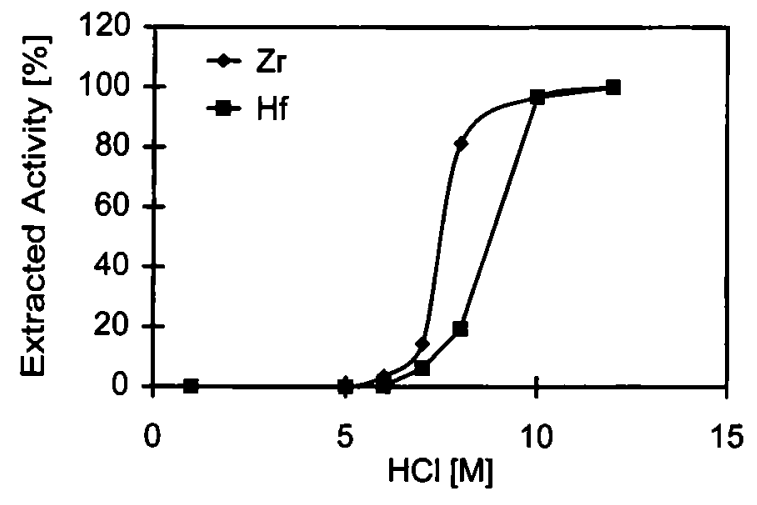

Fig. 2. Percent extraction of $\mathrm{Zr}$ and $\mathrm{Hf}\left({ }^{95} \mathrm{Zr}\right.$ and $\left.{ }^{175} \mathrm{Hf}\right)$ from $\mathrm{HCl}$ solutions into undiluted TBP/Voltalef (1:5).

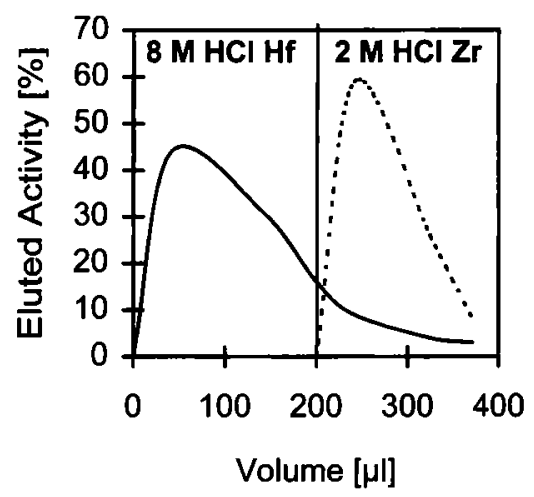

Fig. 3. Elution curves for ${ }^{169} \mathrm{Hf}(8 \mathrm{M} \mathrm{HCl})$ and ${ }^{98} \mathrm{Zr}(2 \mathrm{M} \mathrm{HCl})$ from $1.6 \times 8 \mathrm{~mm}$ columns filled with TBP/Voltalef $(1: 5)$ measured in ARCA II under identical conditions as in the Rf experiments, i.e. the activities were produced on-line and transported to ARCA II by a $\mathrm{He} / \mathrm{KCl}$ jet, dissolved and fed onto the column in $167 \mu 12 \mathrm{M} \mathrm{HCl}$.

Chromatographic separations of $\mathrm{Zr}$ and $\mathrm{Hf}$ on TBP/Voltalef columns of $1.6 \times 8 \mathrm{~mm}$ in ARCA II were studied in $7.5,7.7$, and $8.0 \mathrm{M} \mathrm{HCl}$ after feeding of ${ }^{95} \mathrm{Zr}$ and ${ }^{175} \mathrm{Hf}$ onto the column in $12 \mathrm{M} \mathrm{HCl}$. The best separation was achieved by eluting the $\mathrm{Hf}$ in $8.0 \mathrm{M}$ $\mathrm{HCl}$ followed by stripping the $\mathrm{Zr}$ in $2 \mathrm{M} \mathrm{HCl}$. The elution curves measured with short-lived ${ }^{169} \mathrm{Hf}$ and ${ }^{98} \mathrm{Zr}$ under conditions that were identical to those in the $\mathrm{Rf}$ experiments are shown in Fig. 3. In $200 \mu 1$ of $8 \mathrm{M}$ $\mathrm{HCl}, 75 \%$ of $\mathrm{Hf}$ and no $\mathrm{Zr}$ are eluted. The following elution in $200 \mu \mathrm{l}$ of $2 \mathrm{M} \mathrm{HCl}$ was almost complete for $\mathrm{Zr}(93 \%)$ and removed the remainder of the Hf from the column. The $75 \%$ elution of $\mathrm{Hf}$ in $200 \mu \mathrm{l}$ of $8 \mathrm{M}$ $\mathrm{HCl}$ is the expected result according to the relation

$$
K_{d}=\left\{\frac{100}{\% \mathrm{aq}}-1\right\} \frac{V_{\mathrm{aq}}}{V_{\mathrm{org}}}
$$

where \%aq is the percentage of activity eluted into the aqueous phase $(75 \pm 5 \%), V_{\mathrm{aq}}$ is the volume of the aqueous phase $(200 \mu \mathrm{l}$ minus $35 \mu \mathrm{l}$ which is the void volume in ARCA including the free column volume [14]), and $V_{\text {org }}$ is the volume of the organic phase $(1.03 \mu 1)$. According to Eq. (2), the resulting $K_{d}=53_{-13}^{+15}$. This is consistent with the $K_{d}$ determined
Table 1. Correlated ${ }^{261} \mathrm{Rf}^{257}$ No mother-daughter $\alpha$-decays

\begin{tabular}{cccc}
\hline \multicolumn{5}{c}{ Hf-fraction $(8 \mathrm{M} \mathrm{HCl})$} \\
\hline$E\left(\alpha_{1}\right) / \mathrm{MeV}$ & $t\left(\alpha_{1}\right) / \mathrm{s}^{\mathrm{a}}$ & $E\left(\alpha_{2}\right) / \mathrm{MeV}$ & $t\left(\alpha_{2}\right) / \mathrm{s}^{\mathrm{b}}$ \\
\hline 8.07 & 98.9 & 8.24 & 10.4 \\
8.22 & 57.5 & 8.02 & 47.1 \\
\hline \multicolumn{4}{c}{ Zr-fraction $(2 \mathrm{M} \mathrm{HCl})$} \\
\hline 8.13 & 71.8 & 8.12 & 58.0 \\
8.12 & 14.8 & 8.12 & 61.1 \\
8.23 & 76.8 & 8.23 & 25.0 \\
\hline
\end{tabular}

a After begin of counting.

b After mother decay.

in batch experiments for $\mathrm{Hf}$ in $8 \mathrm{M} \mathrm{HCl}$ which is $64_{-8}^{+9}$. It was the goal of the subsequent Rf experiments to determine the $K_{d}$ for $\mathrm{Rf}$ in $8 \mathrm{M} \mathrm{HCl}$ in the same way, i.e. from the number of ${ }^{261} \mathrm{Rf} \alpha$-decays in the Hfand the $\mathrm{Zr}$-fraction.

1140 experiments were conducted with ${ }^{261} \mathrm{Rf}$ in ARCA II. The collection time of the aerosol was $90 \mathrm{~s}$. Because of the risk of absorption of group- 4 elements on fluoride containing material [10] a titanium slider was used to collect the activity in 798 of the experiments. As some $\mathrm{Ti}$ was dissolved by the $\mathrm{HCl}$, the resulting evaporated samples for $\alpha$-spectroscopy were not ideally weightless resulting in an $\alpha$-energy resolution of $100 \mathrm{keV}$. In the subsequent 342 experiments, the Ti slider was replaced by a Kel-F slider. Apparently, this did not reduce (by absorption) the rate at which ${ }^{261} \mathrm{Rf}$ was observed. Due to the resolution of $100 \mathrm{keV}$ in the majority of the experiments, a window of $\alpha$-energies from 8.0 to $8.65 \mathrm{MeV}$ was used to search for $\alpha$-decays of ${ }^{261} \mathrm{Rf}(8.29 \mathrm{MeV})$ and its daughter ${ }^{257} \mathrm{No}(8.22,8.27,8.32 \mathrm{MeV})$ and for time-correlated pairs of mother-daughter decays. The upper end of the energy window, $8.65 \mathrm{MeV}$, was selected because of the recent observation of another decay branch of ${ }^{261} \mathrm{Rf}$ at $8.52 \mathrm{MeV}$ [15]. The counting time for each sample was $6 \mathrm{~min}$.

$5 \alpha \alpha$-mother daughter correlations in the above mentioned energy window were registered, see Table 1. The fact that they are observed both in the Hf-fraction (2) and in the $\mathrm{Zr}$-fraction (3) points to the fact that $\mathrm{Rf}$ extraction from $8 \mathrm{M} \mathrm{HCl}$ is somewhere between that of $\mathrm{Hf}$ and $\mathrm{Zr}$.

In order to determine the probability for random correlations, the total number of $\alpha$-decays in the considered energy window, 20 in the Hf-fraction and 16 in the $\mathrm{Zr}$-fraction, have to be considered and their time distribution has to be randomly redistributed by a Monte-Carlo method. In the Hf fraction, the number of random correlations predicted in 10000 simulations is 0.15 , which means that the probability for the two observed correlations to be random is $1.0 \%$. In the $\mathrm{Zr}$ fraction, the predicted number of random correlations is 0.08 and the resulting probability for the three observed correlations to be random is $0.008 \%$. Thus, the 


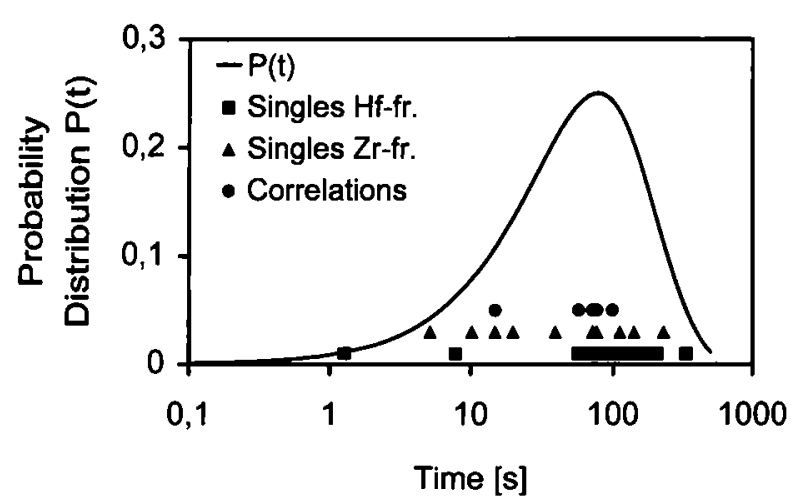

Fig. 4. Time distribution of the $\alpha$-decays of ${ }^{261} \operatorname{Rf} f^{257}$ No. The solid line is the probability distribution for $t_{1 / 2}=78 \mathrm{~s}$.

observed correlations of ${ }^{261} \mathrm{Rf}$ - and ${ }^{257} \mathrm{No}$-decays are likely to be true correlations. The half-life of $28 \mathrm{~s}$ resulting from the ${ }^{257}$ No decay times in Table 1 is in good agreement with the accepted value of $26 \mathrm{~s}$. The time distribution of the singles and correlated events is shown in Fig. 4 together with the probability distribution for $\alpha$-decays of ${ }^{261} \mathrm{Rf}$ with a half-life of $78 \mathrm{~s}$ [13]. The fact that some of the decays are due to the daughter, $26-\mathrm{s}{ }^{257} \mathrm{No}$, i.e. occur with a certain delay, is ignored in this representation of the data.

Before the number of ${ }^{261} \mathrm{Rf}$ - and ${ }^{257} \mathrm{No}$-decays in the Hf- and $\mathrm{Zr}$-fraction can be used to calculate the $K_{d}$ for $\mathrm{Rf}$, a careful consideration of background is in order. The background in the considered energy window from cosmic rays, electronic noise, etc., was determined in long counting intervals before and after the Rf experiment as 7.1 events in all detectors during the complete counting time of $1140 \times 6 \mathrm{~min}$. The number of decays of ${ }^{211 \mathrm{~m} P o}$ at $8.305 \mathrm{MeV}(0.25 \%)$ was determined from the $a$-line of ${ }^{211 \mathrm{~m}} \mathrm{Po}$ at $7.28 \mathrm{MeV}$ (91\%) which consisted of 27 events in the sum spectrum of all samples. This adds 0.074 events to the background. Thus, a total of 7.17 background events distributed over all samples, i.e. 3.59 for both the Hfand the $\mathrm{Zr}$-fraction have to be subtracted. This results in $20-3.59=16.41$ events for the Hf-fraction and $16-3.59=12.41$ events in the $\mathrm{Zr}$-fraction. The latter number has to be further corrected because the start of counting of the $\mathrm{Zr}$-fraction was $24 \mathrm{~s}$ later than the start of counting of the Hf-fraction. With a half-life of $78 \mathrm{~s}$ for ${ }^{261} \mathrm{Rf}$ taken into account, this increases the number of events in the $\mathrm{Zr}$-fraction to 15.36 . With these numbers, we arrive at the conclusion that $51.7+-8.9 \%$ of the Rf was eluted into the Hf-fraction in $(200-35=$ 165) $\mu 18 \mathrm{M} \mathrm{HCl}$. With Eq. (2), this gives a $K_{d}$ value of $150_{-46}^{+64}$. This value shows that $\mathrm{Rf}$ extraction into TBP is larger than that of Hf and we obtain the extraction sequence $\mathrm{Zr}>\mathrm{Rf}>\mathrm{Hf}$, see Fig. 5 , with the $K_{d-}$ values 1180,150 , and 64 for $\mathrm{Zr}, \mathrm{Rf}$, and $\mathrm{Hf}$, respectively.

If one looks at the percentage of extraction in the work of Czerwinski et al. [8] which is $\sim 60 \%$ for $\mathrm{Rf}$ and $\sim 25 \%$ for $\mathrm{Hf}$, and the percentage extraction in the present work, $\sim 52 \%$ for $\mathrm{Rf}$ and $\sim 25 \%$ for $\mathrm{Hf}$, the

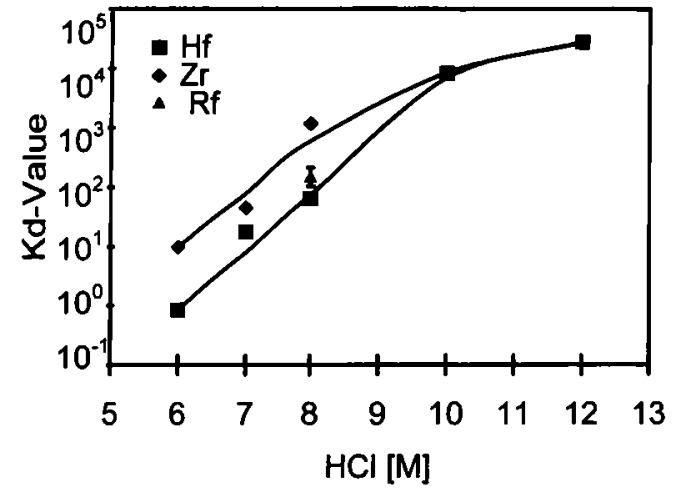

Fig. 5. $K_{d}$-values of $\mathrm{Zr}$ and $\mathrm{Hf}$ in the system undiluted $\mathrm{TBP} /$ Voltalef- $\mathrm{HCl}$ (same experimental data as in Fig. 2) with the $K_{d}$ for $\mathrm{Rf}$ at $8 \mathrm{M} \mathrm{HCl}$.

data, at first sight seem to be consistent. However, one has to consider the extremely different ratios of volumes of the aqueous and organic phase used in [8] $(10 \mu \mathrm{l}: 20 \mu \mathrm{l})$ and in the present work $(165 \mu \mathrm{l}: 1.03 \mu \mathrm{l})$, to realize that significant differences exist: With Eq. (2), $K_{d}$-values for $\mathrm{Rf}$ and $\mathrm{Hf}$ resulting from the data in [8] results as $\sim 0.75$ and $\sim 0.17$, respectively. In order to compare these with our data the dependence of the equilibrium constant of Eq. (1) on the TBP concentration in the organic phase needs to be taken into account. For $x=2$ in Eq. (1), one obtains a ratio of distribution coefficients

$$
\frac{K_{d 1}}{K_{d 2}}=\frac{[\mathrm{TBP}]_{1}^{2}}{[\mathrm{TBP}]_{2}^{2}}
$$

for two different experiments using different TBP concentrations. The TBP concentration in [8] was $0.25 \mathrm{M}$; for our liquid-liquid extraction data in Fig. 1 the TBP concentration was $1.8 \mathrm{M}$. Thus, the $K_{d 1}(0.25 \mathrm{M})=$ 0.17 for $\mathrm{Hf}$ in $8 \mathrm{M} \mathrm{HCl}$ in [8] translates into an expected $K_{d 2}(1.8 \mathrm{M})=8.8$ in our work, while the measured value is 49 , i.e. different by a factor of $\sim 6$. This means that the $K_{d}$-value in $8 \mathrm{M} \mathrm{HCl}$ for $\mathrm{Hf}$ and most probably also that for $\mathrm{Rf}$ in [8] are significantly too low.

The $K_{d}$-value of 49 in $8 \mathrm{M} \mathrm{HCl}$ for $\mathrm{Hf}$ in our liquid-liquid extraction data could be used to predict the $K_{d}$-value in $8 \mathrm{M} \mathrm{HCl}$ for $\mathrm{Hf}$ for undiluted TBP which is $3.65 \mathrm{M}$. Using the same second-order dependence on TBP concentration as in Eq. (3), we expect a $K_{d}$-value of 201 for undiluted TBP, while the measured value is 64 . This indicates that the $K_{d}$-values in undiluted TBP are relatively low because of aggregation effects as mentioned in the introduction.

\section{Discussion}

The newly established $K_{d}$-values and extraction sequence of the group- 4 elements into TBP, $\mathrm{Zr}>\mathrm{Rf}>$ $\mathrm{Hf}$, is at variance with earlier results and conclusions concerning $\mathrm{Hf}$ and $\mathrm{Rf}$ extraction into TBP $[8,10]$. Apparently, both the original $\mathrm{Hf}$ and the Rf results [8] were falsified by losses due to absorption of these ele- 
ments on teflon surfaces. This remained unobserved as only one phase, the TBP phase, was assayed for the decay of ${ }^{169} \mathrm{Hf}$ and ${ }^{261} \mathrm{Rf}$. In the present chromatographic experiment, the distribution of the group-4 elements between TBP and $8 \mathrm{M} \mathrm{HCl}$ was studied by determining the amount of activity in the $8 \mathrm{M} \mathrm{HCl}$ phase and by stripping the remainder of the activity from the organic phase with $2 \mathrm{M} \mathrm{HCl}$. This ensures complete recovery of the activity and results in reliable $K_{d}$ values. The present work shows that $\mathrm{Rf}$ behaves intermediate between $\mathrm{Hf}$ and $\mathrm{Zr}$ near the threshold in $\mathrm{HCl}$ molarity where extraction of $\mathrm{Hf}$ and $\mathrm{Zr}$ increases steeply, i.e. around $8 \mathrm{M} \mathrm{HCl}$. It is probably extracted as $\mathrm{RfCl}_{4} \cdot 2 \mathrm{TBP}$.

The conclusion in [10], i.e. Rf (like Ti) does not extract as well as $\mathrm{Zr}$ and $\mathrm{Hf}$ because it hydrolyses more easily than $\mathrm{Zr}$ and $\mathrm{Hf}$, cannot be maintained.

Also, the statement in [10] that "these results tend to confirm the results" of the experiments on sorption of $\mathrm{Zr}, \mathrm{Hf}$, and $\mathrm{Rf}$ on cobalt ferrocyanide surfaces [9] is misleading as it relies on data [8] which are outrun. Furthermore, the above statement obviously mixes two independent processes: "hydrolysis of cations" and "hydrolysis of complexes". In 6-12 M HCl, the distribution of chloride complexes between the organic and aqueous phases depends on the hydrolysis of the chloride complexes. In the sorption experiments [9], performed at $\mathrm{HCl}$ concentrations below $4 \mathrm{M}$, the sorption depends on the hydrolysis of cations.

Hydrolysis of cations [16] is generally expressed by the following equilibria

$$
\begin{aligned}
& \mathrm{xM}\left(\mathrm{H}_{2} \mathrm{O}\right)_{w^{+}}^{\mathrm{z}+}+ \mathrm{y} \mathrm{OH}^{-} \Leftrightarrow \mathrm{M}_{\mathrm{x}} \mathrm{O}_{\mathrm{u}}(\mathrm{OH})_{\mathrm{y}-2 \mathrm{u}}\left(\mathrm{H}_{2} \mathrm{O}\right)_{\mathrm{w}}^{(\mathrm{x} z-\mathrm{y})+} \\
&+\left(\mathrm{xw}^{0}+\mathrm{u}-\mathrm{w}\right) \mathrm{H}_{2} \mathrm{O} .
\end{aligned}
$$

In another way, each step in the formation of a series of mononuclear species can be described as a successive loss of protons

$$
\mathrm{M}\left(\mathrm{H}_{2} \mathrm{O}\right)_{\mathrm{w}}^{\mathrm{z}} \Leftrightarrow \mathrm{MOH}\left(\mathrm{H}_{2} \mathrm{O}\right)_{\mathrm{w}-1}^{(\mathrm{z}-1)+}+\mathrm{H}^{+} .
$$

Hydrolysis of complexes, in turn, is expressed generally by the following equilibria:

$$
\begin{aligned}
\mathrm{xM}\left(\mathrm{H}_{2} \mathrm{O}\right)_{w^{0}}^{\mathrm{z} t}+ & \mathrm{yOH}^{-}+\mathrm{aA}^{-} \\
& \Leftrightarrow \mathrm{M}_{\mathrm{x}} \mathrm{O}_{\mathrm{u}}(\mathrm{OH})_{\mathrm{y}-2 \mathrm{u}}\left(\mathrm{H}_{2} \mathrm{O}\right)_{\mathrm{w}} \mathrm{A}_{\mathrm{a}}^{(\mathrm{xz}-\mathrm{y}-\mathrm{a})+} \\
& +\left(\mathrm{xw}^{0}+\mathrm{u}-\mathrm{w}\right) \mathrm{H}_{2} \mathrm{O}
\end{aligned}
$$

Reactions (4) and (6) are different processes and their constants are not related: Cations of $\mathrm{Zr}$ are more hydrolyzed $\left(\log K_{11}=0.295\right)$ than cations of $\mathrm{Hf}$ $\left(\log K_{11}=0.146\right)$ [16], while in the extraction experiments, $\mathrm{Zr}$ is better extracted (less hydrolyzed) than $\mathrm{Hf}$ $[10$, this work]. The same is observed for group 5 where $\mathrm{Nb}$ as a cation is more hydrolyzed than $\mathrm{Ta}$ [16], while in the extraction from $\mathrm{HCl}$ solutions, $\mathrm{Nb}$ is better extracted than Ta due to the stronger hydrolysis of the latter [17]. Also electrodialysis experiments [18] show that $\mathrm{Nb}$ forms anionic complexes in $\mathrm{HCl}$ solutions at lower chloride concentrations than $\mathrm{Ta}$, with those of Ta being more hydrolyzed.
It is tempting to discuss the hydrolysis of cations further. In [9], the lower sorption of Rf on cobalt ferrocyanide surfaces was interpreted as Rf being more hydrolyzed than $\mathrm{Zr}$ and $\mathrm{Hf}$. Assuming the same coordination number $(\mathrm{CN})$ of water molecules for all the group-4 elements, this result would contradict the simple ionic model of hydrolysis which implies that cations of the same charge and larger ionic radii are less hydrolyzed. As an explanation of the seemingly stronger hydrolysis of $\mathrm{Rf}$, a decrease in $\mathrm{CN}$ from 8 to 6 has been supposed [9]. This was thought to be caused by the destabilization of the $6 d_{5 / 2}$ orbitals preventing them from being involved in the chemical bonding with valence orbitals of oxygen of water molecules. From the energies of the spin-orbit (SO) splitted $6 d_{3 / 2}$ and $6 d_{5 / 2}$ orbitals of Rf (see Fig. 3 in Ref. [19]) we see that they are by $2 \mathrm{eV}$ lower than the $7 p$ orbitals. Being subjected to a indirect relativistic effect, their SO splitting is not large (about $1 \mathrm{eV}$ ) and less than that of the $7 p$ orbitals. The SO splitting of the $6 p$ orbitals of $\mathrm{Pb}$ (about $1.5 \mathrm{eV}$ ) is even larger, nevertheless both $6 p_{1 / 2}$ and $6 p_{3 / 2}$ orbitals are involved in bonding. The smaller energy difference for the valence orbitals of $\mathrm{Rf}$ cannot be a reason for not attaining a $s p^{3} d^{4}$ hybridization requiring in addition to the $6 d_{3 / 2}$ also the $6 d_{5 / 2}$ orbitals. On the contrary, it is known [20] that increasing relativistic effects in the transactinides result in a close spacing of all the valence levels (the difference between $7 s, 6 d$, and $7 p$ levels is just $1-$ $2 \mathrm{eV}$ for $\mathrm{Rf}$ ), so that the higher $\mathrm{CN}$ are easily obtained. For comparison: for $\mathrm{Ge}, \mathrm{Sn}$, and $\mathrm{Pb}$, the difference between the valence $s$ and $p$ levels is on the order of 8-10 eV and, for example, for $\mathrm{Sn}$, the promotion energy $s^{2} p^{2} \rightarrow s p^{3}$ is about $5 \mathrm{eV}$, and still the $s p^{3}$ or $s p^{3} d^{2}$ hybridization is reached giving $\mathrm{CN}=4$ or 6 . In addition, all MO calculations of the light transactinides [21] have shown the $6 d_{5 / 2}$ orbitals to participate even stronger in chemical bonding than $6 d^{3 / 2}$ orbitals since they are more expanded due to relativistic effects and have a higher overlap with the valence orbitals of ligands.

As an example for "relativistically" reduced lower $\mathrm{CN}$, in Ref. [9], complexes of $\mathrm{Hg}$ are mentioned. The main feature of compounds of $\mathrm{Au}, \mathrm{Hg}$, or $\mathrm{Tl}$ having a preference for $\mathrm{CN}=2$ is a particular shortening and strengthening of two bonds. In [22], the reason for this was said to be a large $s-p$ separation and a preference for $s p$ hybridization instead of $s p^{n}$ hybridization where $n>1$. One should not forget that here the question is about a particular stabilization of the $6 s^{2}$ pair and the contraction of the corresponding valence shell due to both relativistic effects and shell-structure effects (completion of the $d$ shell). Even if the bond lengths are generally independent of the shell contraction, in case of $\mathrm{Au}, \mathrm{Hg}$, and $\mathrm{Tl}$ such a relativistic contraction of the $6 s$ shell (and of the $s p_{1 / 2}$ hybrid orbitals as well since the $6 p_{1 / 2}$ orbitals are also relativistically contracted) influences the metal-ligand bond lengths making them short. The further one goes away from the "gold maximum" the longer get the bond distances. Another 
explanation of the special case of $\mathrm{Hg}$, by the way, was a small $5 d-6 s$ distance [23] which may also be attributed largely to relativistic effects. If to apply all these arguments to the separation of the $6 d_{3 / 2}-6 d_{5 / 2}$ orbitals in the case of $\mathrm{Rf}$, it is hard to see which advantage the $s p^{3} d_{3 / 2}^{2}$ hybridization for $\mathrm{CN}=6$ should have over $s p^{3} d_{3 / 2}^{2} d_{s / 2}^{2}$ for $\mathrm{CN}=8$ in terms of bond strengths or bond lengths. Both $d_{3 / 2}$ and $d_{5 / 2}$ orbitals are diffuse and destabilized.

Generally, the $s-p$ separation alone cannot explain the advantage of the linear hybridization. In $\mathrm{Tl}, 6 s-$ $6 p_{1 / 2}$ separation is 0.238 a.u. and $6 s-6 p_{3 / 2}=0.273$ a.u., and $\mathrm{Pb}$ is not much larger: $6 s-6 p_{1 / 2}=0.291$ a.u., and $6 s-6 p_{3 / 2}=0.305$ a.u. [24]. Nevertheless, $\mathrm{Tl}\left(\mathrm{H}_{2} \mathrm{O}\right)_{6}^{3+}$ is known to have a preference to form two stronger bonds to two of the water ligands while $\mathrm{Pb}^{4+}$ is not.

In Ref. [25], the electronic structures of hexahydrated divalent $\mathrm{Zn}^{2+}, \mathrm{Cd}^{2+}$, and $\mathrm{Hg}^{2+}$ ions have been calculated. The effect of strengthening of two particular metal-oxygen bonds in $\mathrm{Hg}\left(\mathrm{H}_{2} \mathrm{O}\right)_{6}^{2+}$ was found to be stipulated by the action of the second-order JahnTeller effect, resulting in the distortion of the original $\mathrm{O}_{\mathrm{h}}$ structure. The latter was due to a smaller value of the energy gap $\Delta E$ between occupied and vacant levels (with an excitation energy of $7.2 \mathrm{eV}$ ) in the $\mathrm{Hg}$ complex in comparison with $\mathrm{Zn}$ and $\mathrm{Cd}$ (with excitation energies of 12.5 and $11.7 \mathrm{eV}$, respectively), allowing mixing of these levels by the $E_{g}$ vibrational mode. The second-order Jahn-Teller effect is known to be active when the energy gap $\Delta E$ between occupied and the first vacant MO is small so that the "softening" criterium of the system is fulfilled: $\Delta E<2 a^{2} / K$ where $K$ is the force constant and $a=d V / d R_{0}$ with $V$ being the molecular potential and $R_{0}$ the internuclear distance at the point of equilibrium. The second order Jahn-Teller effect was also found to be responsible for the strengthening of two bonds in the $\mathrm{Tl}\left(\mathrm{H}_{2} \mathrm{O}\right)_{6}^{3+}$ compound [26].

One could try to apply these arguments to $\operatorname{Rf}\left(\mathrm{H}_{2} \mathrm{O}\right)_{8}^{4+}$ vs. $\operatorname{Rf}\left(\mathrm{H}_{2} \mathrm{O}\right)_{6}^{4+}$. One could suppose a loss of two water molecules due to some distortion of the regular $\mathrm{O}_{\mathrm{h}}$ structure. Calculations of different compounds of $\mathrm{Rf}$ and other transactinides [21] have shown that $\Delta E$ increases from the $4 d$ to $5 d$ and further to $6 d$ elements so that it is hard to foresee a smaller $\Delta E$ in $\mathrm{Rf}\left(\mathrm{H}_{2} \mathrm{O}\right)_{8}^{4+}$ than that in $\mathrm{Zr}\left(\mathrm{H}_{2} \mathrm{O}\right)_{8}^{4+}$ and $\mathrm{Hf}\left(\mathrm{H}_{2} \mathrm{O}\right)_{8}^{4+}$ (though the calculations of these complexes would be desirable). In addition, recent calculations [27] of the electronic structure of $\mathrm{M}\left(\mathrm{H}_{2} \mathrm{O}\right)_{6}^{5+}(\mathrm{M}=\mathrm{Nb}, \mathrm{Ta}, \mathrm{Pa}$, $\mathrm{Ha}$ ) and study of their hydrolysis have shown that the hydrolysis of these cations follows the sequence $\mathrm{Nb}>$ $\mathrm{Ta}>\mathrm{Ha}>\mathrm{Pa}$ being for $\mathrm{Nb}, \mathrm{Ta}$, and $\mathrm{Pa}$ in agreement with experiment [16]. It turned out that the reason for this is a predominant change in the electrostatic metalligand interaction when water molecules are replaced by $\mathrm{OH}^{-}$groups. Since groups 4 and 5 are very similar, see $[21,23]$, one can foresee a similar sequence in group 4: $\mathrm{Zr}>\mathrm{Hf}>\mathrm{Rf}$. Thus, the weaker sorption of
$\mathrm{Rf}^{4+}$ on cobalt ferrocyanide surfaces [9] can hardly be explained by its stronger hydrolysis.

Let us come back to the present work in which complex formation has been studied for $\mathrm{HCl}$ concentrations between 6 and $12 \mathrm{M}$. Complex formation and the hydrolysis of complexes are competing processes characterized by the following simplified version of Eq. (6):

$$
\mathrm{M}(\mathrm{OH})_{x} \mathrm{Cl}_{4-x}+x \mathrm{HCl} \Leftrightarrow \mathrm{MCl}_{4}+x \mathrm{H}_{2} \mathrm{O} \text {. }
$$

To predict the equilibrium constant for Eq. (7), one has to consider the difference in total energies of the (partially) hydrolyzed species on the left hand side of the equilibrium that are not extracted and of the extractable $\mathrm{MCl}_{4}$. (It is assumed that the $\mathrm{OH}^{-}$containing species will not extract into the organic phase because of the strong hydrogen bonding interaction between $\mathrm{OH}^{-}$ and $\mathrm{H}_{2} \mathrm{O}$.) This can be done by quantum-chemical calculations, presently using the DS DV method $[29,21]$, allowing calculations of the differences in the Coulomb and covalent parts of the binding energy separately. Calculations for $\mathrm{Zr}, \mathrm{Hf}$, and $\mathrm{Rf}$ are still to be done, but one can already draw qualitative conclusions based on a parallel study of the hydrolysis of chloro complexes of the group 5 elements $\mathrm{Nb}, \mathrm{Ta}, \mathrm{Pa}$, and $\mathrm{Ha}$ [30]. For group 5, the order of complex formation described by equilibria similar to the reaction (7) in 4-12 M HCl solutions was found to be $\mathrm{Pa}>\mathrm{Nb}>$ $\mathrm{Ha}>\mathrm{Ta}$. The reason for this are dominant differences in the Coulomb part of the energy of reaction when $\mathrm{OH}^{-}$groups are replaced by $\mathrm{Cl}^{-}$anions.

Earlier calculations [28] of $\mathrm{MCl}_{4}(\mathrm{M}=\mathrm{Zr}, \mathrm{Hf}$, and $\mathrm{Rf}$ have shown that the compounds are very similar, which can also be supposed for $\mathrm{M}(\mathrm{OH})_{\mathrm{x}} \mathrm{Cl}_{4-\mathrm{x}}$. Knowing the analogy in the electronic structure of the halides and oxyhalides of groups 4 and 5 [21, 30], one can postulate the same order of complex formation $\mathrm{Zr}>\mathbf{R f}>\mathrm{Hf}$ according to Eq. (7) as it was found for the corresponding group 5 elements. For both group 4 and 5 , such a sequence is in full agreement with experimental data for $\mathrm{Zr}$ and $\mathrm{Hf}$, and $\mathrm{Nb}$ and $\mathrm{Ta}$, respectively, showing that compounds of the $5 d$ elements are more hydrolyzed than those of the $4 d$ elements and are hence less extracted. For these elements, the hydrolysis of complexes is of opposite order to the order of hydrolysis of the cations which underlines that these processes should not be mixed.

In summary, we have established an extraction sequence of the group- 4 elements from $8 \mathrm{M} \mathrm{HCl}$ into TBP, $\mathrm{Zr}>\mathrm{Rf}>\mathrm{Hf}$, which is in qualitative agreement with theoretical considerations on the competition between hydrolysis of the chloro complexes in the aqueous phase and extraction of the chloro complexes into the organic phase.

\section{Acknowledgments}

The authors are indebted to the Division of Chemical Sciences of the Office of Basic Energy Science of U.S. 
Department of Energy for making the ${ }^{248} \mathrm{Cm}$ available through the transplutonium element production facilities at the Oak Ridge National Laboratory.

We wish to thank Dr. P. Schmelzbach and his crew of the Philips Cyclotron of the Paul Scherrer Institute for providing the ${ }^{18} \mathrm{O}$ beam which produced element 104 for the first time in Switzerland. This work was sponsored by the BMBF under contract 03-HE3 MAI-7.

\section{References}

1. Markus, Y., Kertes, A. S.: Ion Exchange and Solvent Extraction of Metal Complexes, Wiley-Interscience, New York 1969, p. 741.

2. McKay, H. A., Scargill, D., Wain, A. G.: Gmelins Handbuch der Anorganischen Chemie, Transurane A1, II, SpringerVerlag, Berlin 1975, p. 308.

3. McKay, H. A., Sowerby, M. G., Bustraan, M., Monitzaan, J., van Dalen, A., Verkerk, B.: The Separation and Recycling of Actinides, European Atomic Energy Community Rep., EUR-5801e, Brussels 1977.

4. Coleman, C. F., Leuze, R. E.: Some Milestone Solvent Extraction Processes at the Oak Ridge National Laboratory, J. Tenn. Acad. Sci. 53, 102 (1978).

5. Silva, R. J., Harris, J., Nurmia, M., Eskola, K., Ghiorso, A.: Inorg. Nucl. Chem. Lett. 6, 871 (1970).

6. Hulet, E. K., Lougheed, R. W., Wild, J. F., Landrum, J. H., Nitschke, J. M., Ghiorso, A.: J. Inorg. Nucl. Chem. 42, 79 (1980).

7. Czerwinski, K. R., Gregorich, K. E., Hannink, N. J., Kacher, C. D., Kadkhodayan, B. A., Kreek, S. A., Lee, D. M., Nurmia, M. J., Türler, A., Seaborg, G. T., Hoffman, D. C.: Radiochim. Acta 64, 23 (1994).

8. Czerwinski, K. R., Kacher, C. D., Gregorich, K. E., Hamilton, T. M., Hannink, N. J., Kadkhodayan, B. A., Kreek, S. A., Lee, D. M., Nurmia, M. J., Türler, A., Seaborg, G. T., Hoffman, D. C.: Radiochim. Acta 64, 29 (1994).

9. Bilewicz, A., Siekierski, S., Kacher, C. D., Gregorich, K. E., Lee, D. M., Stoyer, N. J., Kadkhodayan, B. A., Kreek, S. A., Lane, M. R., Sylwester, E. R., Neu, M. P., Mohar, M. F., Hoffman, D. C.: Radiochim. Acta 75, 121 (1996).

10. Kacher, C. D., Gregorich, K. E., Lee, D. M., Watanabe, Y., Kadkhodayan, B. A., Wierczinski, B., Lane, M. R., Sylwes- ter, E. R., Keeney, D. A., Hendricks, M., Stoyer, N. J., Yang, J., Hsu, M., Hoffman, D. C., Bilewicz, A.: Radiochim. Acta 75, 127 (1996).

11. Szeglowski, Z., Bruchertseifer, H., Domanov, V. P., Gleisberg, B., Gusiewa, L. J., Hussonnois, M., Tikhomirowa, G. S., Zvara, I., Oganessian, Yu. Ts.: Radiochim. Acta 51, 71 (1990).

12. Kacher, C. D., Gregorich, K. E., Lee, D. M., Watanabe, Y., Kadkhodayan, B. A., Wierczinski, B., Lane, M. R., Sylwester, E. R., Keeney, D. A., Hendricks, M., Hoffman, D. C.: Radiochim. Acta 75, 135 (1996).

13. Kadkhodayan, B. A., Ph. D. Dissertation, University of California, Berkeley, California, LBL-33961 (1993); Kadkhodayan, B. A. et al.: Radiochim. Acta 72, 169 (1996).

14. Schädel, M., Brüchle, W., Jäger, E., Schimpf, E., Kratz, J. V., Scherer, U. W., Zimmermann, H. P.: Radiochim. Acta 48, 171 (1989).

15. Hofmann, S., Ninov, V., Hessberger, F. P., Armbruster, P., Folger, H., Münzenberg, G., Schött, H. J., Popeko, A. G., Yeremin, A. V., Saro, S., Janik, R., Leino, M.: Z. Phys. A354, 229 (1996).

16. Baes, Jr., C. F., Mesmer, R. E.: The Hydrolysis of Cations, John Wiley, New York (1976).

17. Scherff, H.-L., Herrmann, G.: Z. Elektrochem. 64, 1022 (1960).

18. Nabivanets, B. I.: Russ. J. Inorg. Chem. 7, 1428 (1962); Nabivanets, B. I.: Russ. J. Inorg. Chem. 11, 1470 (1966).

19. Glebov, V. A., Masztura, L., Nefedov, V. S., Zhuikov, B. L.: Radiochim. Acta 46, 117 (1989).

20. Fricke, B.: Struct. Bond. 21, 89 (1975); Pershina, V., Sepp, W.-D., Fricke, B., Rosen, A. J.: Chem. Phys. 96, 8367 (1992).

21. Pershina, V.: Chem. Rev. 96, 1977 (1996)

22. Pyykkö, P., Desclaux, J.-P.: Acc. Chem. Res. 12, 276 (1979); Pyykkö, P.: Chem. Rev. 88, 563 (1988).

23. Orgel, L. E.: J. Chem. Soc. 4186 (1958).

24. Desclaux, J. P.: At. Data Nucl. Data Tables 12, 311 (1973).

25. Probst, M. M.: J. Mol. Struct. (Theochem) 253, 275 (1992).

26. Blixt, J., Glaser, J., Mink, J., Persson, I., Persson, P., Sandström, M. J.: Am. Chem. Soc. 117, 5089 (1995).

27. Pershina, V.: Radiochim. Acta 80, 65 (1998).

28. Pershina, V., Fricke, B.: J. Phys. Chem. 98, 6468 (1994).

29. Rosen, A., Ellis, D. E.: J. Chem. Phys. 62, 3039 (1975); Bastug, T.: doctoral thesis, University of Kassel, 1994.

30. Pershina, V.: Radiochim. Acta 80, 75 (1998). 